\title{
O IMPACTO DE UM MANUAL \\ DE ORIENTAÇÕES NA ADESÃO \\ AO TRATAMENTO MEDICAMENTOSO \\ DA HIPERTENSÃO ARTERIAL \\ E QUALIDADE DE VIDA DOS IDOSOS
}

\author{
Gisele Santana Santos ${ }^{1}$ \\ Eduardo Filoni² \\ Vera Lúcia dos Santos Alves ${ }^{3}$
}

resumo

O envelhecimento traz aspectos sociais e físicos que devem ser considerados pelos profissionais da saúde para adequar o dia a dia dos pacientes à rotina medicamentosa. A prevalência da hipertensão arterial sistêmica e a necessidade do consumo regular das medicações de controle interferem diretamente na morbimortalidade dos idosos hipertensos e em sua qualidade de vida. Assim, objetivamos

\footnotetext{
1 Graduada em Enfermagem. Professor da Universidade de Mogi das Cruzes. E-mail: gisa_rose@ hotmail.com.

2 Graduado em Fisioterapia. Doutor em Saúde da Criança e do Adolescente. Coordenador do Curso de Fisioterapia e CST de Radiologia da Universidade Mogi das Cruzes (UMC). E-mail: edufiloni@hotmail.com.

3 Graduada em Fisioterapia. Doutora em Ciências da Saúde. Responsável pelo Serviço de Fisioterapia da Irmandade da Santa Casa de Misericórdia de São Paulo, orientadora dos Programas de Mestrado de Políticas Públicas e Ciências e Tecnologia da Universidade Mogi das Cruzes, e Coordenadora do Instituto de Pesquisa Clínica da Santa Casa de São Paulo. E-mail: fisioterapiasc@uol.com.br.
} 
avaliar a qualidade de vida e a aderência ao tratamento da hipertensão em idosos, analisando o impacto de um manual de orientações para a rotina medicamentosa em idosos hipertensos institucionalizados e de uma comunidade de um município paulista. Foram incluídos 28 idosos alocados em dois grupos: intervenção (receberam as orientações de um manual para o consumo das medicações) e controle (não receberam orientações adicionais na rotina medicamentosa). Todos foram avaliados e reavaliados após dois meses com o teste de Morisky-Green e os questionários WHOQOL-OLD e BREF, sendo observado o aumento da pontuação da primeira pergunta do primeiro teste e da pontução de alguns domínios do questionário OLD após a reavaliação no grupo intervenção. Por fim, foi possível perceber que houve melhora na qualidade de vida dos idosos após terem recebido as orientações do manual proposto.

palavras-chave

Qualidade de Vida. Adesão à Medicação. Hipertensão. Envelhecimento.

\section{Introdução}

As manifestações culturais e o somatório com doenças crônicas daqueles que envelhecem na contemporaneidade são estudados e podem ser identificados com mudanças significativas de hábitos, imagens, crenças e termos utilizados para caracterizar esse período da vida. Além das tradicionais representações que relacionam os momentos mais tardios da vida com a inatividade, surgem hábitos e práticas que associam o processo de envelhecimento ao ato de tomar medicações (SILVA, 2008). Essa imagem está associada ao diagnóstico de alguma doença nessa população que acarreta necessidade de medicações de uso diário. Dentre elas, a hipertensão arterial sistêmica (HAS) é prevalente e pode ser definida como uma condição clínica multifatorial caracterizada por níveis elevados e sustentados de pressão arterial, que podem levar o indivíduo a alterações funcionais e estruturais dos órgãos-alvo (BASTOS-BARBOSA et al., 2012).

Nos idosos, a hipertensão surge em mais de $60 \%$ da população com a persistência no acompanhamento, sendo fator chave para a redução da morbimortalidade (BASTOS-BARBOSA et al., 2012). O tratamento farmacológico impacta significativamente no prognóstico e na qualidade de vida dos pacientes (MÉNDEZ-CHACÓN; SANTAMARÍA-ULLOA; ROSERO-BIXBY, 2008). A 
adesão ao tratamento medicamentoso para os idosos hipertensos, por si só, já é um desafio afetado por fatores cognitivos, sociais e comportamentais, pois além de aspectos relacionados com o tratamento como efeitos colaterais, há a complexidade do regime que deve ser implantado na rotina diária (STRELEC; PIERIN; MION JUNIOR, 2003). A consonância desses fatores interfere na qualidade de vida (QV), que é definida pela Organização Mundial da Saúde (OMS) como a percepção do indivíduo sobre sua posição na vida, no contexto da cultura e no sistema de valores nos quais ele vive (FLECK et al., 2000).

O que não sabemos é se os pacientes que não aderem ao tratamento já possuem uma qualidade de vida diminuída no momento em que param a medicação ou, ainda, nem iniciaram as medicações, o que poderia interferir diretamente no abandono do tratamento e pode ser suplantado com a padronização de orientações para o uso das medicações (GUSMÃO; MION JUNIOR, 2006). Este estudo, portanto, objetivou verificar a qualidade de vida e a aderência ao tratamento da HAS em idosos, analisando o impacto de um manual de orientações para a rotina medicamentosa em idosos hipertensos institucionalizados e de uma comunidade de Mogi das Cruzes.

\section{Casuística e método}

Neste estudo prospectivo e randomizado realizado no período de junho a dezembro de 2013, foram abordados idosos de ambos os sexos e institucionalizados ou residentes de uma mesma comunidade em Mogi das Cruzes. Todos concordaram em participar da pesquisa ao assinarem o Termo de Consentimento Livre Esclarecido, após a liberação do Comitê de Ética e Pesquisa, sob o Certificado de Apresentação para Apreciação Ética (CAAE) n. 0 06359313.6.0000.5497. Critérios de inclusão: diagnóstico de HAS, idade $\geq 60$ anos, cognitivo preservado com capacidade de responder aos questionários e de entender as orientações do manual. Para os idosos institucionalizados, foi instituído que os mesmos deveriam estar nessa situação há mais de um ano. Critérios de exclusão: uso de medicação psicotrópica, alterações motoras que impossibilitassem as condutas descritas no manual de forma independente e aqueles não precisavam alterar as dosagens de medicamento de uso contínuo no período do estudo.

Dos 44 idosos inicialmente abordados, 13 não entraram na pesquisa por usarem psicotrópicos e um se recusou a assinar o Termo de Consentimento. Consequentemente, foram incluídos 30 idosos divididos em dois grupos por meio de envelopes opacos e selados. Após a randomização, tivemos duas perdas 
(uma de cada grupo), pois um paciente faleceu e outro iniciou tratamento oncológico e teve alteração da medicação inicial. Portanto, foram analisados 28 idosos, alocados em dois grupos:

- Grupo intervenção: 14 idosos (nove da comunidade e cinco institucionalizados), que receberam durante dois meses as orientações de um manual sobre a importância do uso adequado das medicações para controle da pressão arterial;

- Grupo controle: 14 idosos (seis da comunidade e nove institucionalizados), que não receberam nenhuma orientação adicional no período do estudo.

Os dois grupos foram abordados verbalmente e, durante esse contato, eram ressaltados o objetivo e a execução do estudo. Após manifestarem a intenção de participarem, foi agendada a visita para a aplicação dos questionários. Nos idosos institucionalizados, essa visita ocorria na residência de longa permanência (regime aberto) e havia a consulta ao prontuário do idoso. Nos pacientes da comunidade, o diagnóstico da HAS surgia durante a entrevista inicial e a análise dos medicamentos de consumo diário, enquantoa entrevista para a coleta dos dados ocorria na residência do idoso. Todos os 28 pacientes responderam a uma ficha com dados pessoais, ao teste de Morisky-Green e aos questionários WHOQOL-OLD e BREF. Todos os idosos foram orientados a escolher a opção que melhor se encaixava a seu caso dentro das possibilidades de resposta dada pelos instrumentos. O teste de Morisky-Green proporciona a verificação da predisposição do paciente em relação à tomada dos remédios (STRELEC; PIERIN; MION JUNIOR, 2003). O WHOQOL-OLD é um instrumento especifico para os idosos e o BREF é genérico e contém 26 questões. Os dois questionários devem ser aplicados em conjunto e os resultados destas avaliações são dados em escala de $0 \%$ a $100 \%$.

Após a avaliação inicial dos idosos, o grupo intervenção recebeu uma vez por semana, em um período de dois meses, a orientação idealizada pelo manual criado pelos autores da pesquisa sobre a importância do uso correto das medicações para controle da pressão arterial. Esse manual foi elaborado com formas ilustrativas que reforçam as orientações quanto aos horários dos medicamentos, seguindo a prescrição médica. Após dois meses da avaliação inicial, os participantes tanto do grupo intervenção como do controle foram reavaliados, sendo aplicados novamente o teste de Morisky-Green e os questionários WHOQOL-OLD e BREF. A análise estatística foi realizada pelo programa SPSS, sendo utilizados os testes de Mann-Whitney para a análise paramétrica dos questionários OLD e BREF e o teste Exato de Fisher para as medidas não paramétricas do teste de Morisky-Green. O teste de Wilcoxon foi 
aplicado para a análise de possíveis diferenças entre os grupos e o teste de Qui-quadrado para a análise dos dois momentos de avaliação e reavaliação com nível de significância $\leq 0,05$.

\section{Resultados}

O perfil do grupo intervenção apresentou média de idade de 68,00 anos $( \pm 5,25)$, e o controle, de 72,07 anos $( \pm 9,80)$. Não houvediferença na comparação entre os grupos para idade ou qualquer outro parâmetro apresentado na Tabela 1.

Tabela 1 - Quantidade e percentual de distribuição de cada grupo (intervenção e controle) e análise total dos indivíduos (n) para as variáveis de sexo, estado civil, escolaridade, tabagismo, consumo de álcool e prática de atividade física.

\begin{tabular}{|c|c|c|c|c|c|c|c|}
\hline \multirow{3}{*}{ Variável } & \multirow{3}{*}{ Categoria } & \multicolumn{4}{|c|}{ Grupo } & \multirow{2}{*}{\multicolumn{2}{|c|}{ Total }} \\
\hline & & \multicolumn{2}{|c|}{ intervenção } & \multicolumn{2}{|c|}{ controle } & & \\
\hline & & $\mathrm{n}$ & $\%$ & $\mathrm{n}$ & $\%$ & $\mathrm{n}$ & $\%$ \\
\hline \multirow{2}{*}{ Sexo } & Feminino & 11 & 78,60 & 06 & 42,90 & 17 & 60,71 \\
\hline & Masculino & 03 & 21,40 & 08 & 57,10 & 11 & 39,29 \\
\hline \multirow{4}{*}{ Estado civil } & Solteiro & 03 & 21,40 & 05 & 35,70 & 08 & 28,57 \\
\hline & Casado & 10 & 71,40 & 06 & 42,90 & 16 & 57,14 \\
\hline & Separado & 01 & 7,10 & 00 & 0,00 & 01 & 3,57 \\
\hline & Viúvo & 00 & 0,00 & 03 & 0,00 & 03 & 10,71 \\
\hline \multirow{4}{*}{ Escolaridade } & Alfabetizado & 07 & 50,00 & 05 & 35,70 & 12 & 42,86 \\
\hline & $\begin{array}{l}\text { Ensino } \\
\text { fundamental } \\
\end{array}$ & 07 & 50,00 & 06 & 42,90 & 13 & 46,43 \\
\hline & Ensino médio & 00 & 0,00 & 03 & 21,40 & 03 & 10,71 \\
\hline & Ensino superior & 00 & 0,00 & 00 & 0,00 & 00 & 0,00 \\
\hline \multirow{2}{*}{ Fuma } & Sim & 01 & 7,10 & 00 & 0,00 & 01 & 3,57 \\
\hline & Não & 13 & 92,90 & 14 & 100,00 & 27 & 96,43 \\
\hline \multirow{2}{*}{$\begin{array}{l}\text { Consumo de } \\
\text { álcool }\end{array}$} & Sim & 00 & 0,00 & 01 & 7,10 & 01 & 3,57 \\
\hline & Não & 14 & 100,00 & 13 & 92,90 & 27 & 96,43 \\
\hline \multirow{2}{*}{ Atividade física } & Sim & 01 & 7,10 & 01 & 7,10 & 02 & 7,14 \\
\hline & Não & 13 & 92,90 & 13 & 92,90 & 26 & 92,86 \\
\hline
\end{tabular}


Quanto ao questionário BREF na comparação inicial entre os grupos intervenção e controle, não houve diferenças significantes. Apesar de os valores absolutos da pontuação dos domínios deste questionário terem aumentado no grupo intervenção, tendendo à melhora da condição geral do idoso após o período de aplicação das orientações do manual, a significância estatística ocorreu somente para o domínio de qualidade de vida $(p=0,031)$, com aumento da pontuação.

Em relação ao questionário OLD, os domínios de morte/morrer e de intimidade apresentaram $p$ de 0,010 e 0,021, respectivamente, denotando que o grupo controle apresentava menor qualidade de vida no momento da avaliação inicial. Após as orientações, o grupo intervenção apresentou aumento da qualidade de vida somente evidenciada com a significância estatística para o domínio morte/morrer, com $p$ de 0,007 (Gráfico 1), apesar da tendência de melhora em todos os domínios.

Gráfico 1 - Representação gráfica com a média de pontuação total na reavaliação (pós) e avaliação (pré) para todos os domínios do questionário OLD nos 28 pacientes dos grupos controle e intervenção.

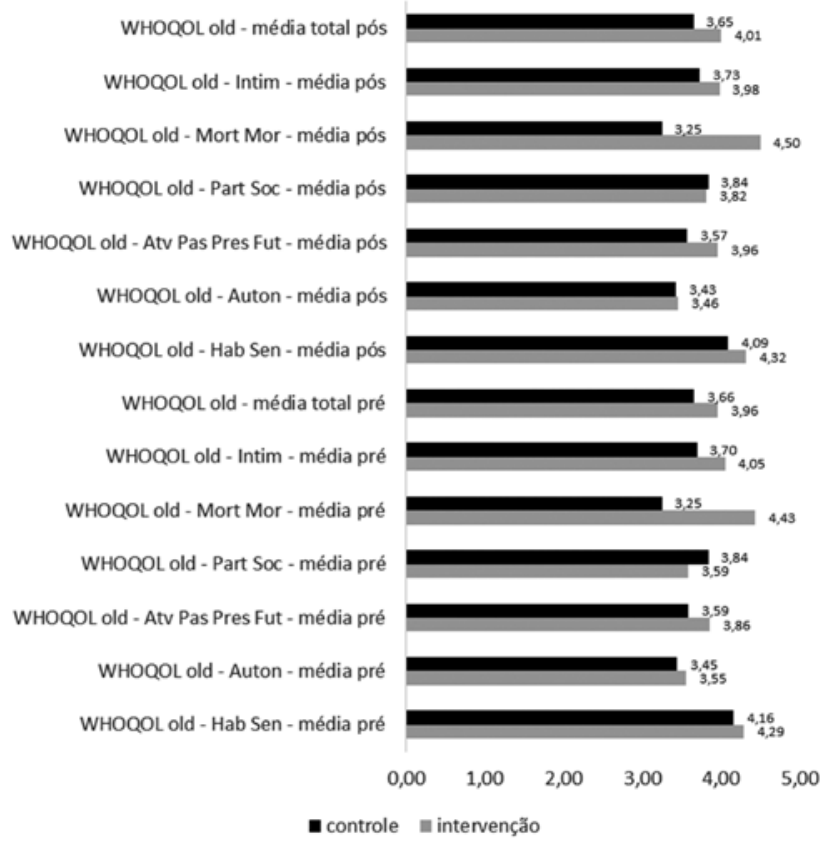


Pós: pós-tratamento; Pré: pré-tratamento; Intim: intimidade; Mort Mor: morte/morrer; Part Soc: participação social; Atv Pas Pres Fut: atividade passada/ presente/futura; Auton: autonomia; Hab Sen: habilidade sensorial.

Para o teste de Morisky-Green, só houve diferença na comparação após a aplicação do manual entre os grupos intervenção e controle na primeira pergunta do teste, que questiona se o paciente esqueceu-se de tomar alguma vez seu remédio, com seis pacientes que esqueciam e passaram a não esquecer após o protocolo $(p=0,014)$ (Gráfico 2).

Gráfico 2 - Comparação gráfica com a média de porcentagem de pontuação pós e préorientações dadas pela aplicação do manual proposto nos grupos intervenção e controle para cada item questionado no teste de Morisky-Green (TGM).

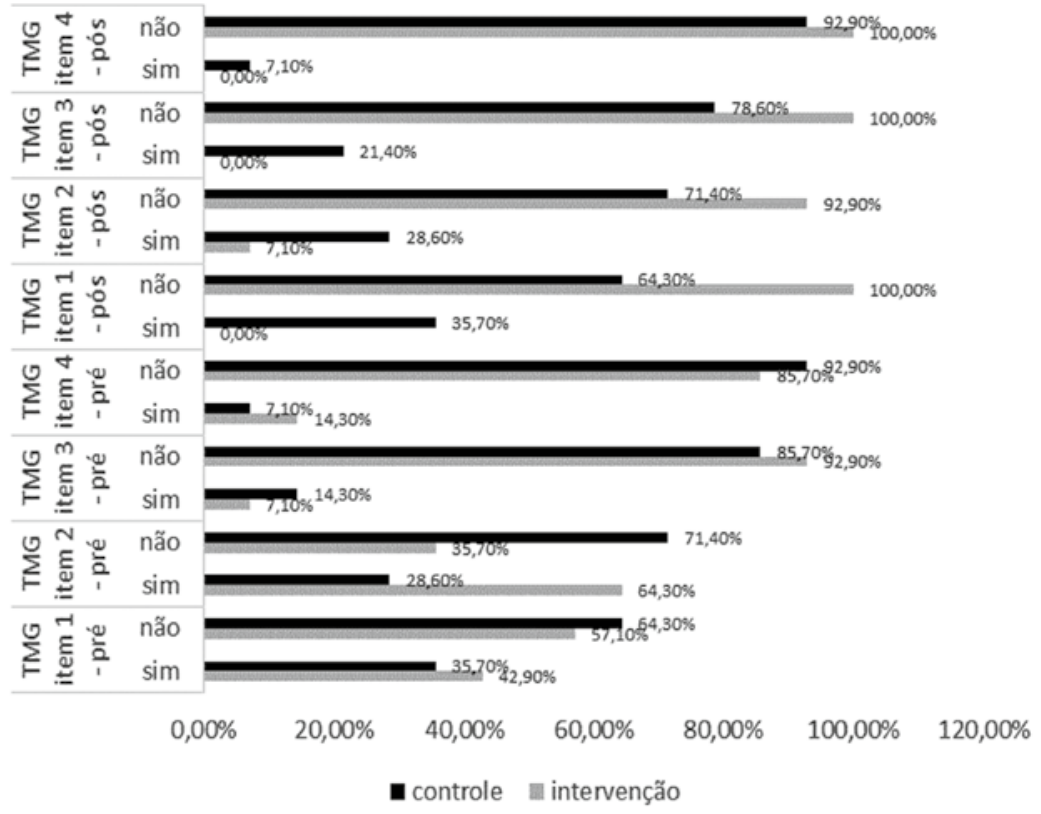

Analisando possíveis diferenças entre os subgrupos de idosos institucionalizados e da comunidade dentro dos grupos de intervenção e controle, os idosos da comunidade (nove participantes na intervenção e seis no controle) demonstraram diferença para o questionário BREF somente no domínio de qualidade de vida. Neste domínio, apresentaram $p$ de 0,007 no momento de 
reavaliação com o subgrupo comunidade dentro do grupo intervenção, apresentando melhor qualidade de vida na comparação.

Já para o questionário OLD, só ocorreu diferença no domínio morte/ morrer com $p$ de 0,032 no momento de reavaliação com o subgrupo comunidade. Isto, mais uma vez, evidencia percepção aumentada de morte avaliada após a intervenção, sem alterações significantes no teste de Morisky Green.

\section{Discussão}

Manuais de orientação para aumento da aderência a tratamento em doenças crônicas têm sido propostos para auxiliar os pacientes a conhecerem de forma mais ampla seu quadro clínico e terem uma atitude mais consciente com o papel ativo no controle de sua saúde (RODINI et al., 2010). O presente estudo mostrou como indivíduos idosos hipertensos respondem diante da utilização de um manual de orientações para o uso de medicamentos, o que reforça a importância da atenção primária e secundária na saúde e seu impacto na qualidade de vida em nossa casuística.

A hipertensão arterial sistêmica (HAS) é uma doença crônica comum na população idosa brasileira, já sendo conhecido seu impacto negativo na ausência de controle medicamentoso adequado, com a história natural da doença demonstrando maior morbimortalidade nos pacientes que não aderem ao tratamento (BASTOS-BARBOSA et al., 2012). Não foi incluída em nosso objetivo a avaliação da incidência de comorbidade associada ao diagnóstico da HAS devido ao intervalo de acompanhamento desta pesquisa ser de apenas dois meses. Porém, mesmo com este pequeno corte, já pudemos observar maior aderência ao tratamento medicamentoso idealizando menor taxa futura de morbidez relacionada com a falta de controle dos níveis de pressão arterial após a implantação do manual de orientações.

Para o manejo dos níveis pressóricos elevados e de suas consequências, são imprescindíveis a identificação e o acompanhamento dos hipertensos pelos serviços de saúde, independente da situação domiciliar ou da capacidade de autocuidado (MENDEZ et al., 2008). Devido a isso, nosso recrutamento incluiu idosos institucionalizados e de uma comunidade alocados de forma cega em um grupo de intervenção e em outro de controle para observarmos se haveria evolução diferente de acordo com a situação domiciliar do idoso. Apesar de observarmos o aumento da pontuação dos dois subgrupos, não houve diferenças significantes entre os grupos após a intervenção. 
Esse resultado talvez possa desmistificar a visão de que o idoso institucionalizado que recebe, em tese, cuidados mais intensos na rotina medicamentosa, por ter acompanhamento técnico, tem qualidade de vida melhor. De acordo com Cianciarrulo (2002), não há resposta simples nesta análise, pois essa população normalmente é contaminada pela imagem preconcebida de isolamento e menor vontade individual, o que não pode ser substituído pelos cuidados técnicos e acabaria igualando os que moram em instituições com os que residem em comunidades.

Sabemos também que em algumas doenças, como a HAS, osteoporose, diabetes e depressão, há alto comprometimento da capacidade funcional e de aspectos sociais, econômicos e demográficos, sendo que esses fatores podem alterar a capacidade do indíviduo de seguir as propostas de tratamento (COTTA et al., 2009). Observando esse quadro, nossa amostra incluiu o perfil dos pacientes idosos, com a inclusão de informações como estado civil, escolaridade, hábito tabagista, consumo de álcool e atividade física para abrangermos uma maior quantidade de informações. Diante disso, podemos traçar um panorama que evidencia melhor o impacto do manual de uso de medicações. Apesar da grande quantidade de informações obtivemos uma amostra homogênea quando pareamos os grupos e levantamos informações de possíveis comportamentos viciosos que podem influenciar na rotina diária do uso da medicação para o controle da HAS e dificultar as comparações entre os grupos.

Notamos, porém, que havia no grupo intervenção mais mulheres, que se diziam casadas, com ensino fundamental completo, sedentárias, não fumantes ou etilistas, perfil próximo ao grupo controle, com a diferença na descrição sendo devida somente ao fato da maioria de homens. Nesse cenário, ficamos contrários a afirmações de Souza e Carvalho (2003), em que a percepção do próprio envelhecimento aumenta com hábitos como o tabagismo e o alcoolismo por se originar de uma forma de fuga, já que em nossa amostra os pacientes negaram possuir esses hábitos.

No planejamento do estudo, imaginávamos que provavelmente haveria diferenças na comparação entre os subgrupos de indivíduos que residiam em instituições quando comparados aos da comunidade. Entretanto, deparamo-nos com um cenário diferente, pois analisando nossos resultados, não encontramos grandes diferenças na comparação inicial entre grupos e subgrupos para os questionários WHOQOL-OLD e BREF, assim como o teste de Morisky Green.

O fato de que não ocorreu significância mesmo com o subgrupo da comunidade, presente tanto no grupo de intervenção como no controle, pode nortear uma visão mais ampla do processo de envelhecer. De acordo com Fleck et al. (2006), o estudo de medidas subjetivas e genéricas de qualidade de vida traz um conjunto de informações que possibilitam traçar perfis e orientam os programas 
de saúde para a criação de medidas sustentáveis para a população de adultos e idosos. Talvez nossa visão esteja contaminada com um processo de envelhecer mais mórbido do que como ele é encarado pelos idosos.

Assim, a criação e indicação para uso sistemático de um manual de orientações para a rotina das medicações pode, nos idosos hipertensos, ratificar a importância dos hábitos saudáveis abandonando ou evitando os prejudiciais. Além disso, é possível diminuir a controversa de informações que surgem de conhecimento empírico, que muitas vezes repassa informações não fidedignas e adequadas, que podem ser padronizadas, ensinadas e seguidas a partir de profissionais da rede de atenção basica à saúde munidos com esses manuais (LESSA, 2006).

De acordo com Souza e Carvalho (2003), avaliar a qualidade de vida envolve conceitos quantitativos e individuais para cada sociedade. Hoje, a abordagem desse conceito precisa levar em consideração as necessidades básicas que promovam o bem-estar subjetivo e social. Por isso, há a defesa do uso de questionários, ainda mais quando a proposta é a de verificarmos a efetividade de uma intervenção em dois momentos em um mesmo indíviduo (SALGADO et al., 2013).

A maioria dos idosos da nossa amostra diz estar satisfeita com a vida na pontuação média dos instrumentos neste estudo randomizado. Encontramos breves diferenças com pouca significância estatística e esse fato pode ser explicado pela instituição de longa permanência que aceitou participar desta pesquisa e é de regime aberto, o que confere a seus moradores a liberdade e a convivência social fora de seu ambiente, possibilitando ter o indicativo positivo para qualidade de vida.

Quando analisamos individualmente o comportamento do teste de Morisky-Green na comparação entre o grupo intervenção e o controle, observamos que o reforço dado pelo manual sobre os horários da tomada de medicamentos pode fazer com que o idoso adquira maior responsabilidade na condução de seu tratamento e no não esquecimento no uso das medicações. Observando a melhora significativa para os que vivem na comunidade na rotina diária do medicamento, ratificamos a necessidade de medidas específicas para esse fim (CASTRO; FUCHS, 2008).

Uma dificuldade encontrada no estudo de Rodini et al. (2010) foi o abandono dos pacientes durante a condução de pesquisas em idosos. Em nosso estudo, os pacientes que entraram nos grupos após a randomização mostraram um comportamento contrário, não abandonando a pesquisa e demonstrando grande atenção e empenho durante as palestras para seguir orientação padronizada através do manual. 
Estimar a aderência ao tratamento com medicação anti-hipertensiva usando métodos diretos ou indiretos tem limitações, não havendo um padrãoouro cuja sensibilidade e especificidade sejam superiores a $80 \%$, mas sabemos que o controle da HAS é relacionado com a pontuação do teste de Morisky Green e que medidas dadas podem auxiliar no controle da doença (BLOCH; MELO; NOGUEIRA, 2008). Assim, acreditamos que a criação e a utilização de manuais podem ser determinantes para a evolução dos pacientes hipertensos. A importância do manual de orientações para hipertensão mostrou significância ao grupo de idosos acompanhados nesta pesquisa, pois quando eles recebem as orientações e a visita do técnico de saúde da atenção primária e têm o controle da doença, há melhora da qualidade de vida (BRITO, 2008), fato que pudemos verificar no período de acompanhamento desses idosos.

\section{Conclusão}

Portanto, esta pesquisa nos permitiu concluír que o manual de orientações para hipertensão mostrou-se significativo. O grupo de idosos acompanhados recebeu as orientações do manual proposto com a cartilha de orientação do controle da doença e apresentaram uma melhora na qualidade de vida. Isto mostra que esse manual é um produto importante para a veiculação nos locais de atendimento aos idosos.

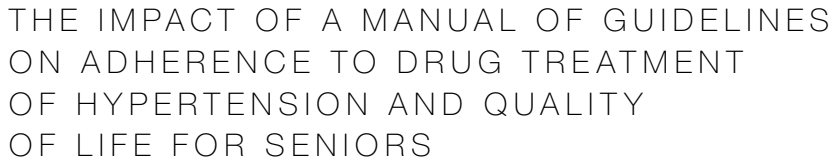

abstract

The aging brings social and physical aspects that should be considered by health professionals to tailor the daily life of patients to drug routine. The prevalence of hypertension and the need for regular use of controller medications interfere directly in the morbidity and mortality of elderly hypertensive patients and their quality of life. Thus, we aimed to evaluate quality of life and adherence to treatment of hypertension in the elderly, analyzing the impact of a manual's guidelines for routine drug in institutionalized elderly hypertensive patients in community of Mogi das Cruzes. They included 28 individuals divided into two groups: intervention (received the guidance of a manual for the consumption 

Morisky-Green test and WHOQOL-OLD and BREF questionnaires. The group monitored of intervention received the manual's guidelines proposed with the guidance booklet of disease control and showed an improvement in quality of life. This shows that this manual is an important product for placement in local elder care.

\section{keywords}

Quality of Life. Medication Adhrence. Hypertension. Aging.

\section{referências}

BASTOS-BARBOSA, Rachel G. et al. Adesão ao tratamento e controle da pressão arterial em idosos com hipertensão. Arquivos Brasileiros Cardiologia, São Paulo, v. 99, n. 1, p. 636-641, jul. 2012.

BLOCH, Katia Vergetti; MELO; André Nascimento de; NOGUEIRA, Armando R. Prevalência da adesão ao tratamento anti-hipertensivo em hipertensos resistentes e validação de três métodos indiretos de avaliação da adesão. Cadernos de Saúde Pública, Rio de Janeiro, v. 24, n. 12, p. 2979-2984, dez. 2008.

BRITO, Fausto. Transição e desigualdades sociais no Brasil. Revista Brasileira de Estudos de População, São Paulo, v. 25, n. 1, p. 5-26, jan./jun. 2008.

CASTRO, Mauro Silveira; FUCHS, Flávio Danni. Abordagens para aumentar a adesão ao tratamento em pacientes com hipertensão arterial de difícil controle. Revista Brasileira de Hipertensão, Rio de Janeiro, v. 15, n. 1, p. 25-27, mar. 2008.

CIANCIARULLO, Tamara Iwanow. Compreendendo a família no cenário de uma nova estratégia de saúde. In: CIANCIARULLO, Tamara Iwanow et al. (Org.). Saúde na família e na comunidade. São Paulo: Robe Editorial, 2002. p. 17-37.

COTTA, Rosângela Minardi Mitre et al. Perfil sociossanitário e estilo de vida de hipertensos e/ou diabéticos, usuários do Programa de Saúde da Família no município de Teixeiras, MG. Ciência \& Saúde Coletiva, Rio de Janeiro, v. 14, n. 4, p. 1251-1260, jul./ago. 2009.

FLECK, Marcelo Pio de Almeida et al. Aplicação da versão em português do instrumento abreviado de avaliação da qualidade de vida "WHOQOL-bref". Revista de Saúde Pública, São Paulo, v. 34, n. 2, p. 178-183, abr. 2000.

Development and validation of the Portuguese version of the WHOQOL-OLD module. Revista de Saúde Pública, São Paulo, v. 40, n. 5, p. 785-791, out. 2006.

GUSMÃO, Josiane Lima de; MION JUNIOR, Décio. Adesão ao tratamento - conceitos. Revista Brasileira de Hipertensão, Rio de Janeiro, v. 13, n. 1, p. 23-25, jan. 2006

LESSA, Ínes. Impacto social da não-adesão ao tratamento da hipertensão arterial. Revista Brasileira de Hipertensão, Rio de Janeiro, v. 13, n. 1, p. 39-46, jan. 2006.

MENDES, Antonio da Cruz Gouveia et al. Assistência pública de saúde no contexto da transição demográfica brasileira: exigências atuais e futuras. Cadernos de Saúde Pública, Rio de Janeiro, v. 28, n. 5, p. 955-964, maio 2012. 
MÉNDEZ-CHACÓN, Ericka; SANTAMARÍA-ULLOA, Carolina; ROSERO-BIXBY, Luis. Factors associated with hypertension prevalence, unawareness and treatment among Costa Rican elderly. BMC Public Health, London, v. 8, n. 1, p. 275, Aug. 2008.

RODINI, Fernanda Batista Carvalho et al. Prevenção de incapacidade na hanseníase com apoio em um manual de autocuidado para pacientes. Fisioterapia e Pesquisa, São Paulo, v. 17, n. 2, p. 157-166, abr./jun. 2010.

SALGADO, Teresa et al. Cross-cultural adaptation of the Beliefs about Medicines Questionnaire into Portuguese; São Paulo Medical Journal, São Paulo, v. 131, n. 2 , p. 88-94, Feb. 2013.

SILVA, Luna Rodrigues Freitas. Da velhice à terceira idade: o percurso histórico das identidades atreladas ao processo de envelhecimento. História, Ciências, Saúde, Manguinhos, Rio de Janeiro, v. 15, n. 1, p. 155-168, jan./mar. 2008.

SOUZA, Rafaela Assis de; CARVALHO, Alysson Massote. Programa de Saúde da Família e qualidade de vida: um olhar da Psicologia. Estudos de Psicologia, Natal, v. 8, n. 3, p. 515-523, set./dez. 2003

STRELEC, Maria Aparecida A. Moura; PIERIN, Angela M. G.; MION JUNIOR, Décio. A influência do conhecimento sobre a doença e a atitude frente à tomada dos remédios no controle da hipertensão arterial. Arquivos Brasileiros de Cardiologia, São Paulo, v. 81, n. 4, p. 343-348, out. 2003.

Recebido: 05/09/2014

Aceite Final: 20/11/2015 